\title{
GamField 2.0 - Significant advances to synthetic potential fields data generator
}

\author{
Alessandro Pignatelli ${ }^{1}{ }^{*}$, Iacopo Nicolosi $^{1}$, Roberto Carluccio ${ }^{1}$, Massimo Chiappini ${ }^{1}$ \\ ${ }^{1}$ Istituto Nazionale di Geofisica e Vulcanologia (INGV), Roma, Italy
}

\author{
Article history \\ Received October 20, 2017; accepted February 2, 2018. \\ Subject classification: \\ Potential fields; Matlab; Software; Computer simulation.
}

\begin{abstract}
GaMField is an integrated system tool, released in 2011 and available for free, able to construct and visualize subsurface sources in 3-D space and to compute their gravimetric and magnetic effects. Since its first release, we have received feedback and new features implementation requests from scientists worldwide. According to such requests, significant advances have been introduced into the software to broaden its field of application in potential field analysis and interpretation as described in this paper.
\end{abstract}

\section{Introduction}

GaMField was released in 2011 [Pignatelli et al. 2011] to realize the graphical windows-interactive constructions of complex 3-D synthetic gravimetric and magnetic sources. Such synthetic models are widely used by researchers to verify potential data fields interpretation algorithms and to predict magnetic or gravity response of a given geological model. [Last and Kubic 1983, Guillen and Menichetti 1984, Li and Oldenburg 1996, Fedi and Rapolla 1999, Pignatelli et al. 2007, Nicolosi et al. 2006].

The software can be freely downloaded, after the registration, at the following URL:

$$
\text { http: / / geosoftware.sci.ingv.it }
$$

where a forum has been set-up for GaMField users and for other possible future geophysical software applications. GaMField has been downloaded by hundreds of users and it has got citations since its presentation [Tontini 2012, Speranza et al. 2012, Al-Rahim 2013, Jeshvaghani and Darijani 2014, Speranza and Minelli 2014, Guo et al. 2015, Liu et al. 2015, Montesinos et al. 2016, Pan et al. 2016, Kim and Wessel 2016]. Many researchers have joined the web forum and provided with a large number of comments and suggestions for im- provements. We have collected these useful inputs and decided to broaden GaMField field of applications with the aim to make it available to the scientific community operating in the area of potential field analysis and interpretation.

Basically three main newly developed tools are now implemented:

- use of space vertical cross sections to build up sources;

- a new interactive GUI to simulate topographic effects;

- STL 3D objects files import and export.

Minor additional improvements have been also introduced. GaMField is maintained by INGV (Italian National Institute of Geophysics and Volcanology).

\section{New tools description}

An extensive description of GaMField main principles and its modules can be found in Pignatelli et al. [2011] as well as in GaMField help. In Figure 1 a schematic representation of such modules and their new inter-operative connections is shown.

The basic "GaMField idea" is using magnetic and gravimetric field linearity to model complex sources using a set of uniformly magnetized rectangular prisms. The total intensity field at a given location is given by the sum of each prism field. The user can specifically set up a calculation volume divided into equal rectangular prisms (the source grid). Once such volume has been set up, the user needs to select the prisms to build up a magnetic or a gravimetric source.

In order to select such prisms GaMField package provides ModelBuilder module, shown in Figure 2 [Pignatelli et al. 2011] (see block "ModelBuilder" in Figure 1). 


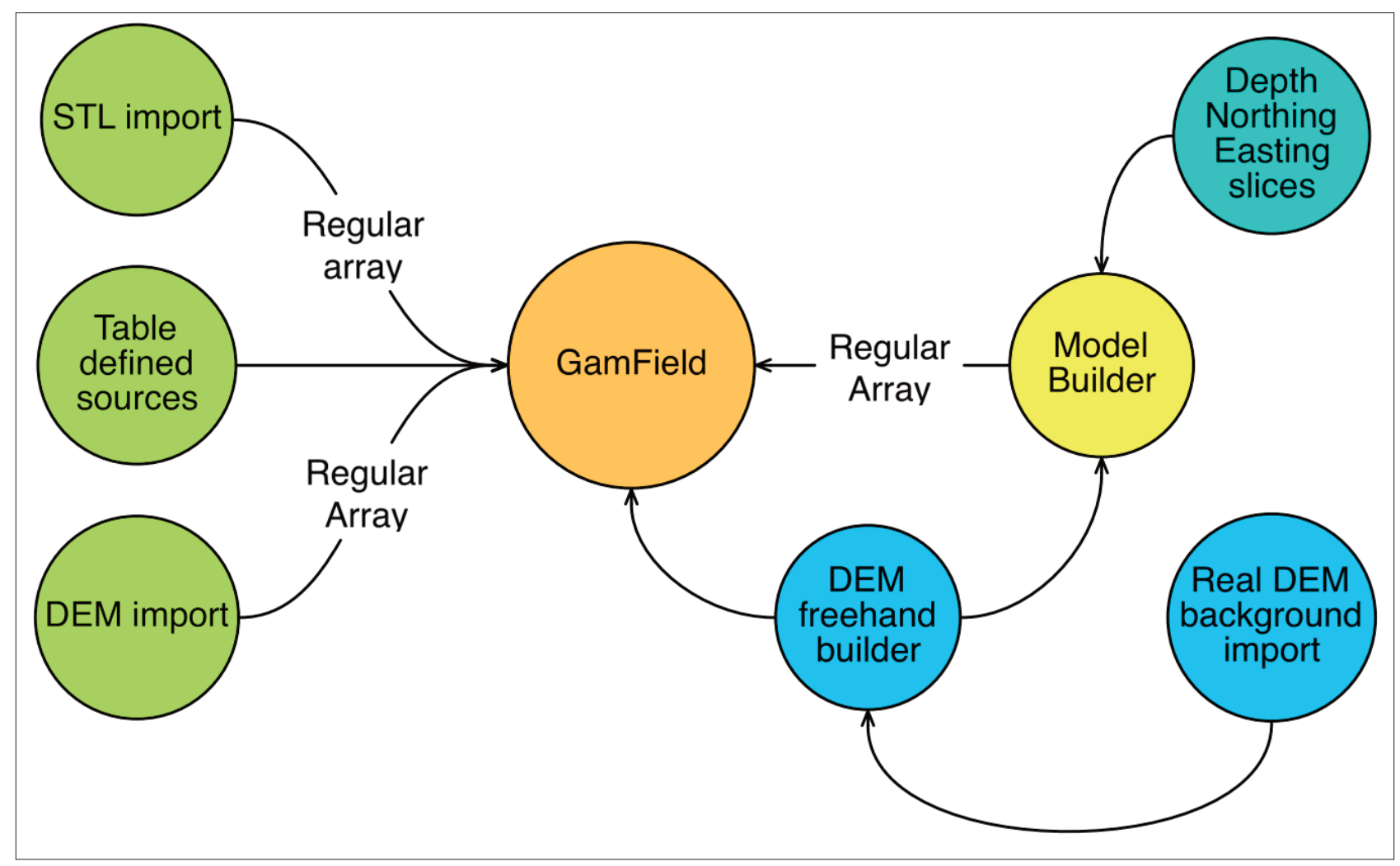

Figure 1. New GaMField modules and their inter-operative connections.

The source grid area is represented by a number of parallel horizontal planes filled with red and blue tiles in Figure 2. Each tile is the top face of a rectangular prism. Each prism extends up to the horizontal plane beneath. At this stage, there are no magnetized prisms and the different colours are there just to identify each prism boundary.

As shown in Figure 2, ModelBuilder allows selecting a horizontal layer by means of the combo box outlined by the green circle.

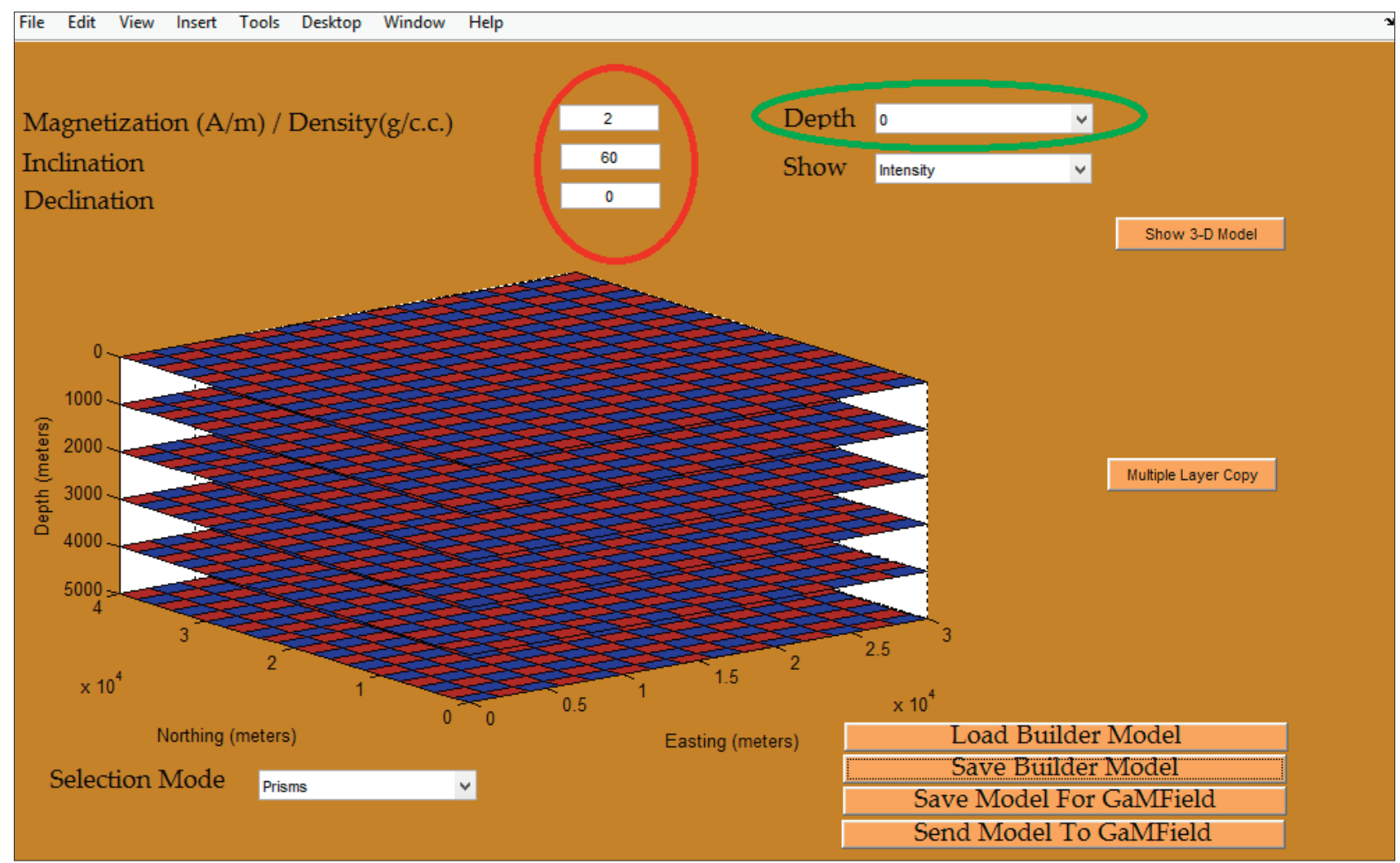

Figure 2. GaMField "ModelBuilder module". 


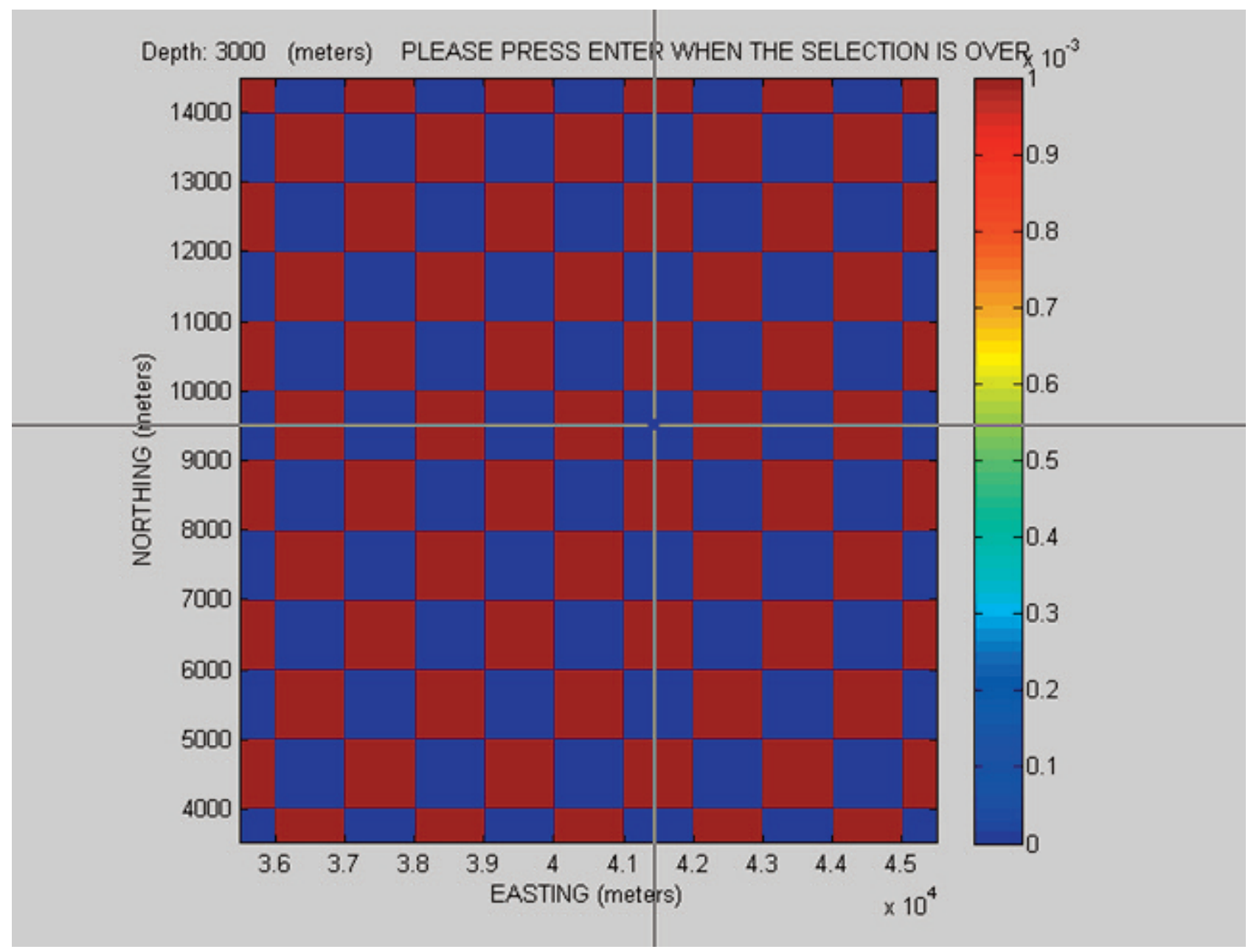

Figure 3. User can "magnetize prisms" of a specific horizontal layer by clicking.

After selecting the horizontal layer, ModelBuilder lets the user select prisms by clicking them and allows to assign separately to each prism a different magnetic angle and magnetization/density values as outlined by the red circle in Figure 2. The assignment is done by clicking on the desidered prisms as shown in Figure 3. Alternatively, selecting "Polygon" option in the "Selection Mode" combo box, the user can also draw a polygon and the software will automatically select all the prisms inside it.

2.1 First upgrading feature: selecting prisms using vertical section

As a lot of geosoftware registered users have pointed out, ModelBuilder module is very efficient when building horizontally extended sources. Unfortunately, it is not so efficient for vertically extended sources. In the new GaMField version, we have added the possibility to choose "Easting layers" or "Northing layers" as shown in Figure 4 (see also block "Depth Northing Easting slices" in Figure 1). As one can see in Figure 5, the new ModelBuilder GUI shows a couple of additional combo boxes (indicated by the A frame) allowing such choice.
The "multiple Easting copy" and "multiple Northing copy" buttons (Figure 5, frame b)) can be used exactly as described in Pignatelli et al. [2011] and in GaMField help for "Multiple layer copy" button.

2.2 Second upgrading feature: topographic source generation

The old version of GaMField was already provided with some topographic simulation tools. A detailed description of such tools can be found in Pignatelli et al. [2011] and in GaMField help. Basically GaMField allows loading a DEM file and automatically build up a set of prisms approximating the loaded DEM in order to compute the corresponding magnetic or gravimetric field (see block "DEM import" in Figure 1). In the new GaMField version, we have introduced a new tool called "DemGenerator", providing the user with the possibility to interactively build a synthetic topographic model "drawing" isolines (see block "DEM freehand builder" in Figure 1). The model produced by this tool is meant to be uniform so inclination, declination and magnetization/density will be the same for the whole built structure. The user could possibly introduce vari- 


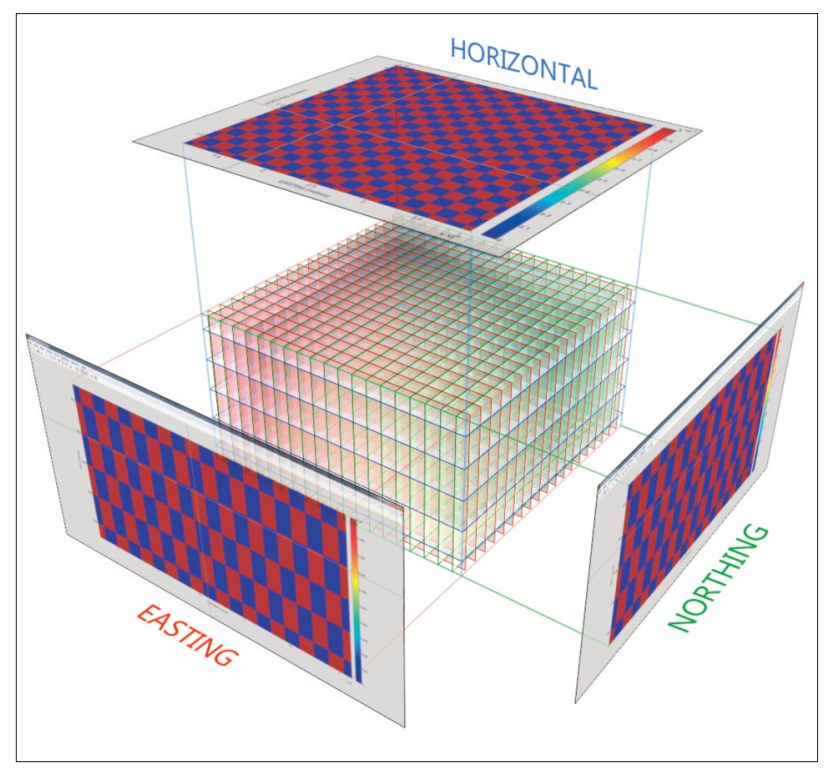

Figure 4. selecting prisms to be magnetized by means of horizontal or vertical sections.

ations using ModelBuilder module. The basic idea of DemGenerator is to define a "top surface" and a "bottom surface" as shown in Figure 6. The two surfaces will define the top and the bottom of the single layer of prisms defining the DEM. Once the two surfaces have been defined, this GaMField module will create uniformly dense/magnetized rectangular prisms with user defined parameters. So basically, the following steps are necessary to define a synthetic DEM:

- define an area where the two surfaces will be built;

- define the prism horizontal sizes;

- define the whole DEM inclination, declination,

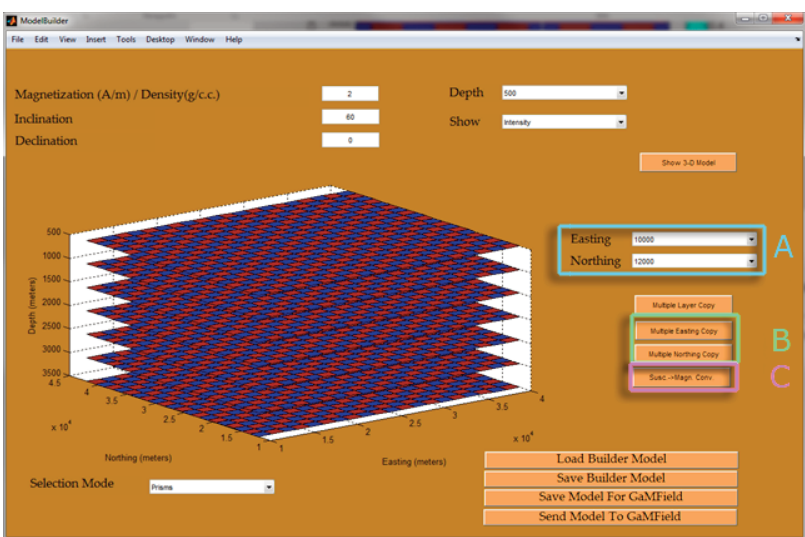

Figure 5. The new ModelBuilder interface. A) The new combo boxes "Easting" and "Northing" B) "Multiple Easting" and "Multiple Northing" layer copy C) Susceptibility to Magnetization conversion button.

magnetization/density values;

- draw top and bottom surfaces by contouring;

- let DemGenerator build the DEM.

DemGenerator can be started by GaMField main GUI by using the menu Topography -> Generate topographic model. The GUI in Figure 7 will be called.

The text boxes defined in "Area details" are needed to define the area where the topography will be generated as well as the prisms size, whereas the section "Dem details" allows the user to set the magnetization/density values of the approximating prisms. After filling in the above text boxes, the user should confirm input parameters validity by clicking "Confirm Parameters" button and then the top and bottom surfaces can be defined. Basically it is needed to write an altitude or depth into "Insert a top Isoline"

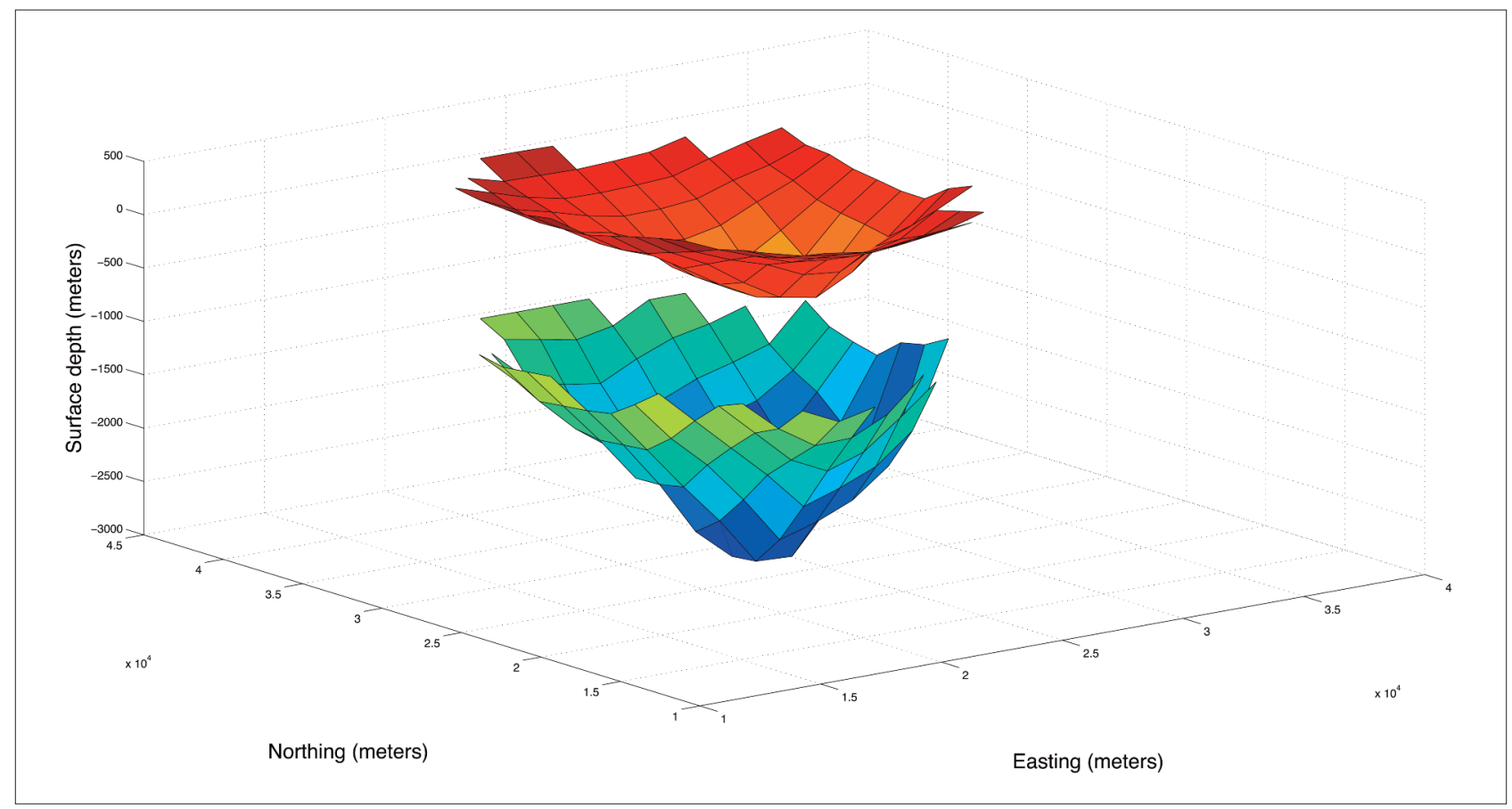

Figure 6. Top and bottom surface. 


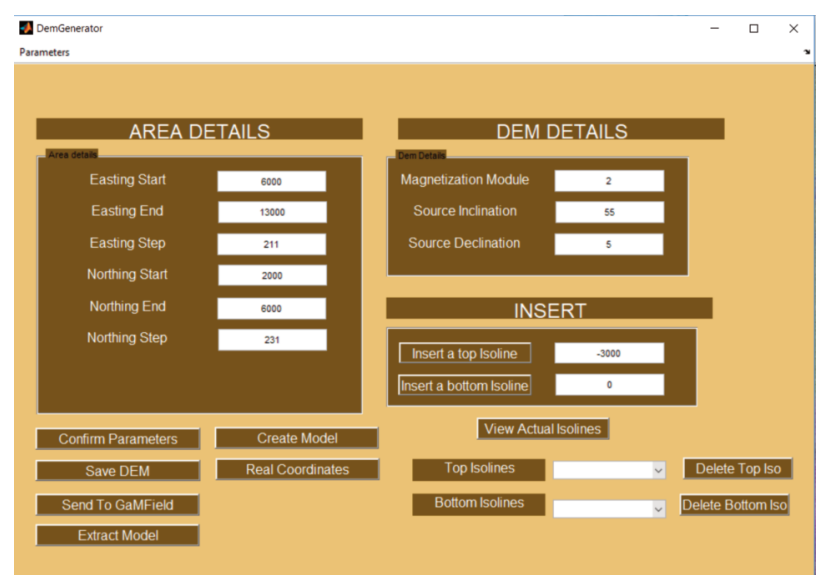

Figure 7. The new tool DemGenerator.

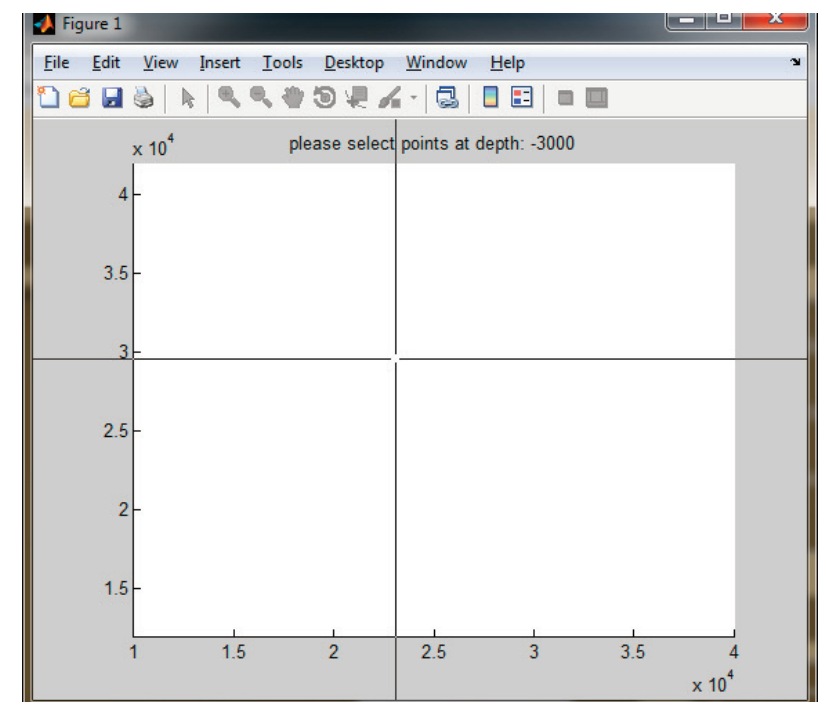

Figure 8. Selecting isolines in DemGenerator module.

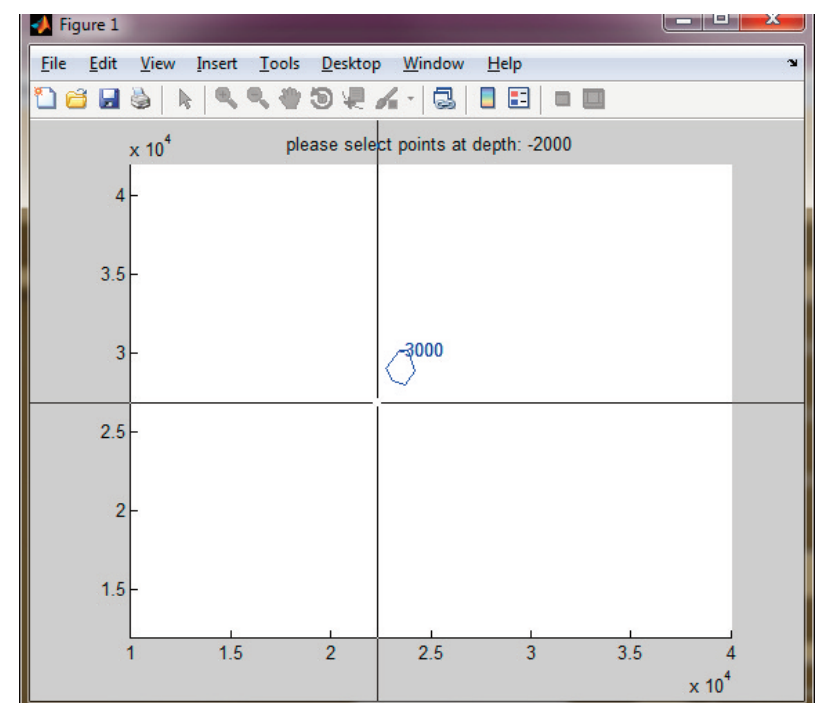

Figure 9. Selecting isolines in DemGenerator module.

or "Insert a bottom Isoline" text box and click to the button on the left of the selected text box. It is important to notice that vertical axis orientation is the same as always used in GaMField, so the positive orientation is downward. After clicking button, DemGenerator

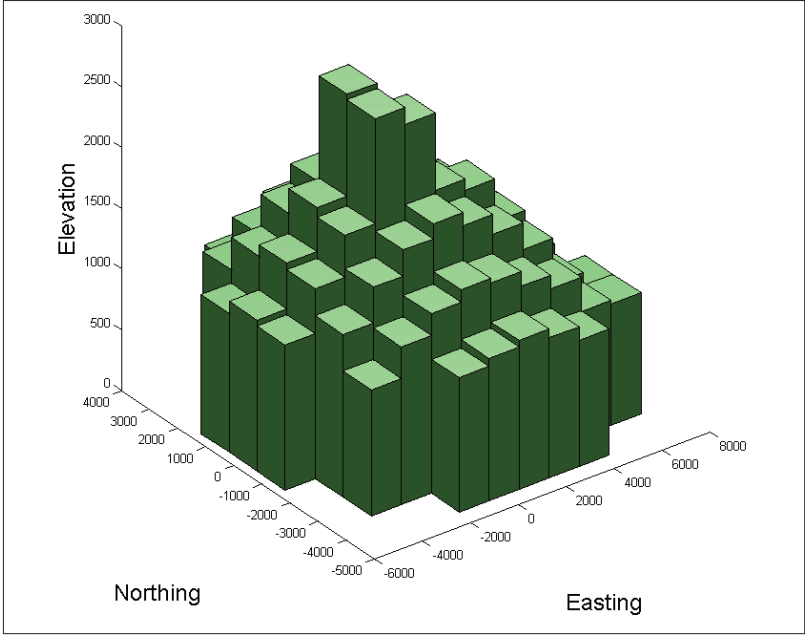

Figure 10. Topographic model generated by DEMGenerator

will pop-up a figure showing the area coordinates and the user can select the altitude/depth isoline by clicking as shown in Figure 8.

One can select as many points as needed and press enter. The software will close the isoline connecting the last clicked point to the first one as shown in Figure 9. When adding the next isolines, the software will show the previous ones as shown in Figure 9.

Once the user has drawn all of the isolines, "Create Model" button needs to be clicked and the software will generate a prisms ensemble approximating the selected pattern (see example shown in Figure 10). Basically, the software will generate a single layer set of prisms where the horizontal coordinates are quantized according to "Area details" parameters and the prisms top and bottom are calculated by a linear interpolation of the isolines defined surface.

The created model will be in GaMField and ModelBuilder format so the user can send it to GaMField by using "Send to GaMField" button or open it into ModelBuilder by means of "Modify with ModelBuilder" button. In the first case, the model will be loaded from GaMField main module as a topographic model, so the computation has to be done by using the menu Topography -> Compute Topographic Magnetic Effects. In the latter case, the user will be prompted to insert the thickness resolution for the model and then the software will open ModelBuilder and the user will be able to modify the source as described in Pignatelli et al. [2011] or in GaMField help. In this case, after sending the final model to GaMField main module, the field computation will be performed by using the menu Compute -> Magnetic Field. Once a topographic model field has been computed, it can be exported into an ascii file by means of the menu File -> Save topographic effects. 


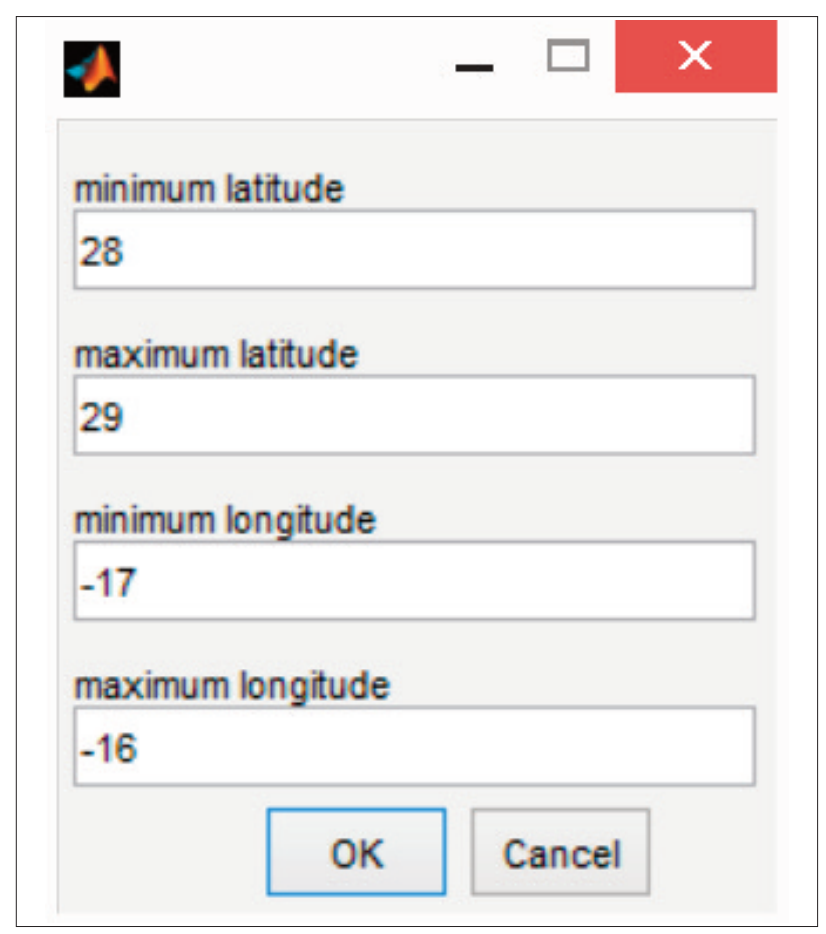

Figure 11. Dialog box appearing when using DEMGenerator "Real Coordinates" button. The right input parameters for Tenerife Island are shown

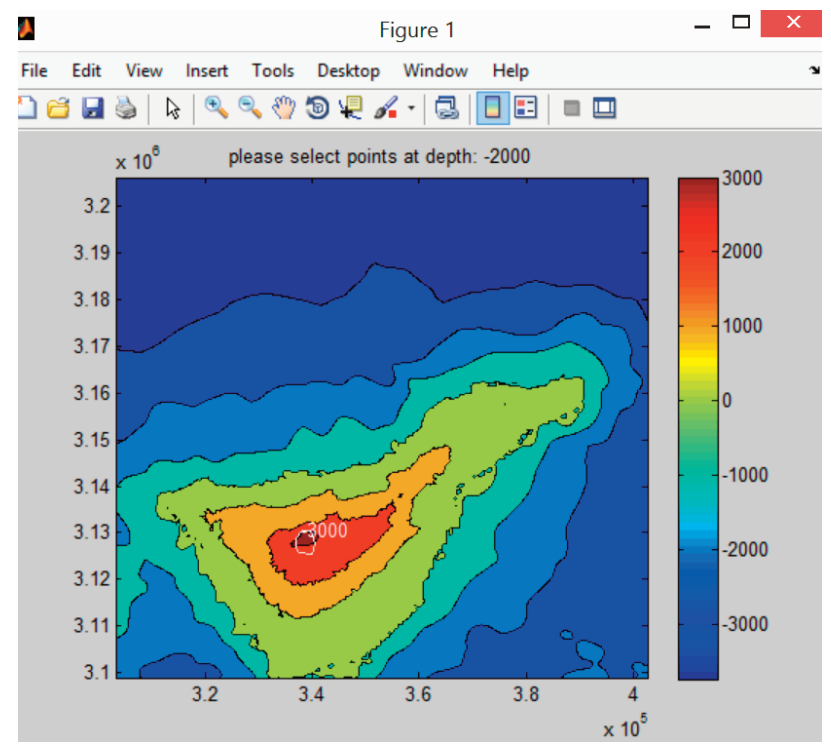

Figure 12. Isolines of Tenerife Island to help user.

\subsubsection{Dem Generator: real coordinates tool}

In order to make Dem generation easier and useful for realistic simulation, in the new GaMField version we have introduced the following possibilities: (i) Build the topographic model using real coordinates; (ii)Visualize real geographical topographies; (iii) Extract a topographic model from real DEM data.

The user has not to search or download any data, he/she just needs an internet connection to use this tool. Let's assume, for example, the user wants to generate a synthetic DEM reproducing as much as possi- ble Tenerife Island Volcanic area or build a generic topographic model in the same zone. He/she just needs to use "Real coordinates" button of DEM Generator module (see Figure 7) and the software will prompt with the dialog box shown in Figure 11 asking for a real rectangular area boundaries specified in geographic coordinates (the ones in Figure 11 refer to Tenerife island).

After inserting such coordinates, the software will automatically take care of changing "Area details" text boxes converting lat/lon coordinates into UTM projection of the selected area to guarantee the selected geographic area as DEM building domain. A message box will be prompted to specify the UTM projection zone. The user will be able to "draw" the isolines on the background shown in the Figure 12:

As stated before, the "real DEM" background will not affect any computation and the DEM creation is exactly how described in the previous paragraph, however, if the user wants to use the downloaded real DEM as the topographic model to compute magnetic or gravimetric field, he/she just needs to push "Extract model" button. All the magnetic/gravimetric parameters chosen in DemGenerator gui will be used to produce the topographic model. The only missing parameter is the bottom, so the user will be asked to input it. The program will exclude from the model every part of the DEM where the top is lower than the bottom chosen by the user.

It is important to state here that this feature uses Mathworks web server mapping service. Basically, matlab runtimes will automatically look for web servers providing DEM data available at request moment. So this feature working depends on third parties servers providing data, so we cannot guarantee a constant and steady correct working. However, if issues occur, we will try to find possible solutions and, if we found them, provide upgrades to the users.

\subsection{Third upgrading feature: STL files import and export}

As reported by many users, even if ModelBuilder module is a very good and efficient tool to simulate magnetic sources, sometimes users are already skilled on different $3 \mathrm{~d}$ modelling software. That's why, in the new version of GaMField, we have introduced the possibility to import standard 3D model format files.

STL is a worldwide standard format for 3D triangular mesh surfaces, largely used in CAD-CAM and 3D printing manufacturing flows. Many editors, ranging from simple freeware to high end production suites, do exist to build and manipulate such meshes. To load an STL file, the user has to use the menu 


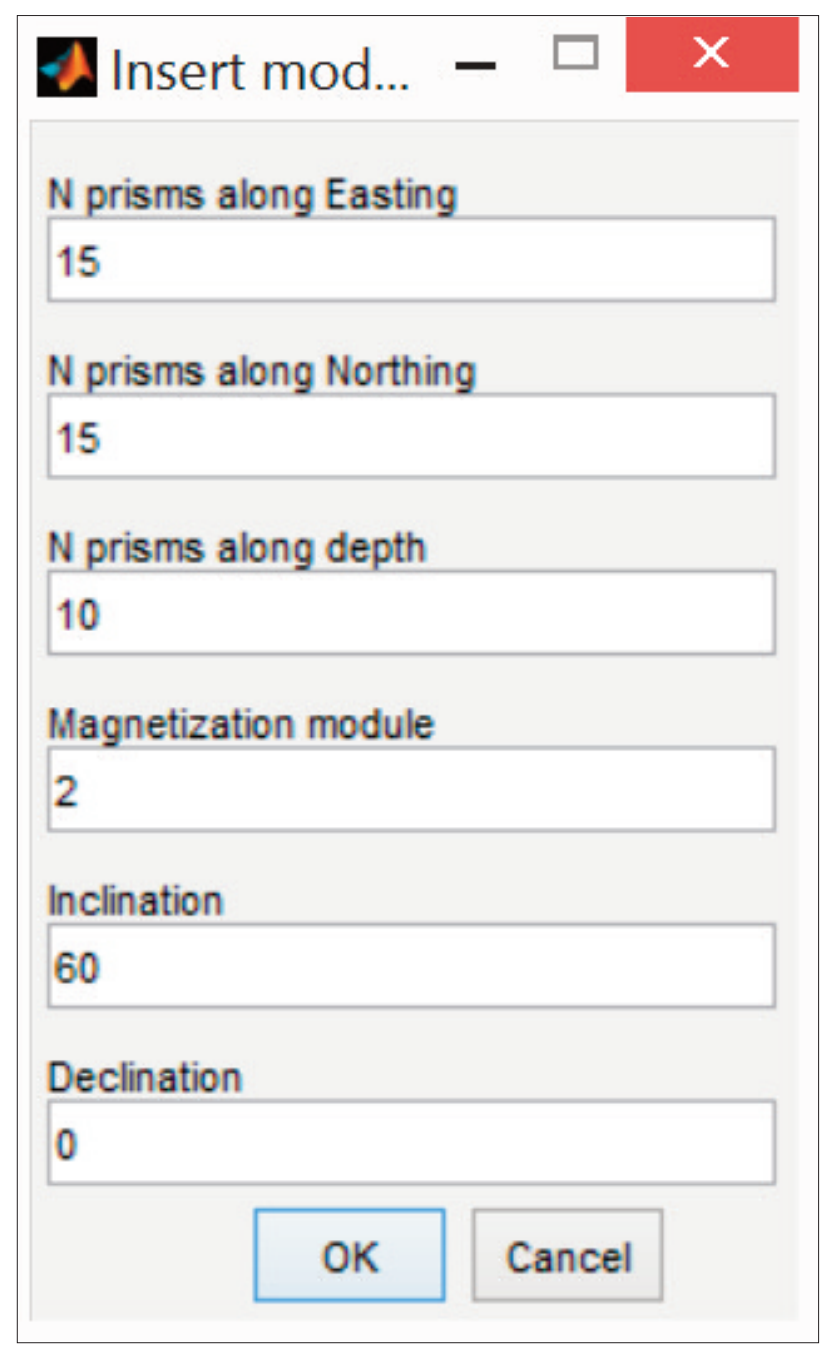

Figure 13. STL import requester window.

File -> Model -> Load STL Model.

Some additional geometric parameters are entered in the dialog window represented in Figure 13.

Once the parameters have been inserted, the STL surface is converted to a set of prisms as any other GaMField model. The user can see the resulting model by means of View -> Built Model menu and perform any other computation. Additionally, it is possible to export a ModelBuilder produced model as STL file using command File -> Model -> Export model STL.

\section{Additional minor changes to GaMField}

\subsection{Magnetization/susceptibility conversion}

Another common requirement asked from geosoftware forum members is about magnetic properties. The old version of GaMField uses both magnetization intensity and direction. Unfortunately, many researchers use magnetic susceptibility. So we have inserted the possibility to immediately convert the susceptibility to magnetization. In the new version of GaMField we have introduced a suite tool to simplify the conversion.
Basically, given the magnetic body susceptibility, GaMField perform the conversion to magnetization parameters by means of the following equations:

$$
M_{T}=M_{I}+M_{R}
$$

where $M_{T}$ is the total magnetization. $M_{I}$ is the source magnetization induced by the external magnetic field and $M_{R}$ is the source remanent magnetization. The remanent magnetization, if wanted, should be introduced by the user by means of the three parameters:

\section{$\left|M_{R}\right|$ : remanent magnetization intensity \\ $I_{R}$ : remanent magnetization inclination \\ $D_{R}$ : remanent magnetization declination}

By means of the previous parameters, it is possible to compute the three cartesian magnetization components:

$$
\begin{aligned}
& M_{R x}=\left|M_{R}\right| \cos \left(I_{R}\right) \cos \left(D_{R}\right) \\
& M_{R y}=\left|M_{R}\right| \cos \left(I_{R}\right) \sin \left(D_{R}\right) \\
& M_{R z}=\left|M_{R}\right| \sin \left(I_{R}\right)
\end{aligned}
$$

$M_{I}$ is the main field induced magnetization. Its cartesian components are given by:

$$
\begin{aligned}
& M_{I x}=\mathrm{B} \chi / \mu \cos \left(I_{I}\right) \cos \left(D_{I}\right) \\
& M_{I y}=\mathrm{B} \chi / \mu \cos \left(I_{I}\right) \sin \left(D_{I}\right) \\
& M_{I z}=\mathrm{B} \chi / \mu \sin \left(I_{I}\right)
\end{aligned}
$$

Where: $B$ is the main magnetic field module, $I_{I}$ is the main magnetic field inclination, $D_{I}$ is the main magnetic field declination, $\chi$ is the source magnetic susceptibility, $\mu$ is a constant given by: $\mu=4 \pi \cdot 10^{-7}$ if the susceptibility is given in S.I.units, $\mu=10^{-7}$ if the susceptibility is given in c.g.s. units. Both unit systems can be used in GaMField.

In GaMField, the user can perform such computation by using the ComputeMagneticParameters module. It can be opened when working with ModelBuilder module by means of button Susc -> Magn Conv button in ModelBuilder module or by "Special" menu in GaMField main GUI. The ComputeMagneticParameters GUI is represented in Figure 14. The user can input all remanent magnetization, susceptibility and main field parameters and then get the magnetization module and angular values.

\subsection{Set survey parameters from model}

One issue identified by Geosoftware users is related to possible source/model missed alignment. Basically, when confirming the input parameters, GaMField checks survey parameters and model parameters inde- 


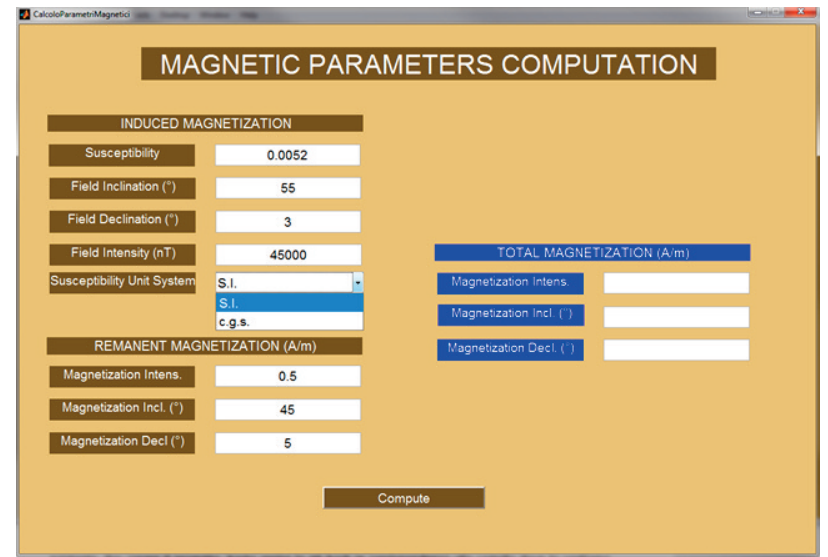

Figure 14. ComputeMagneticParameters module. The user can input all susceptibility, remanent magnetization and main field parameters and then compute the magnetization parameters by using "Compute" button.

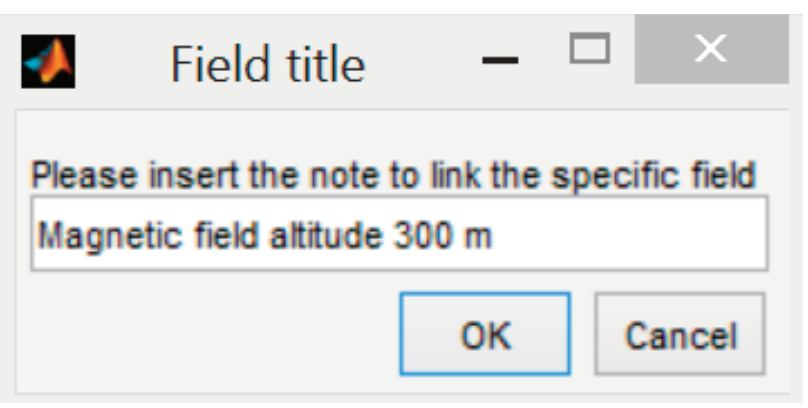

Figure 15. Add history dialog.

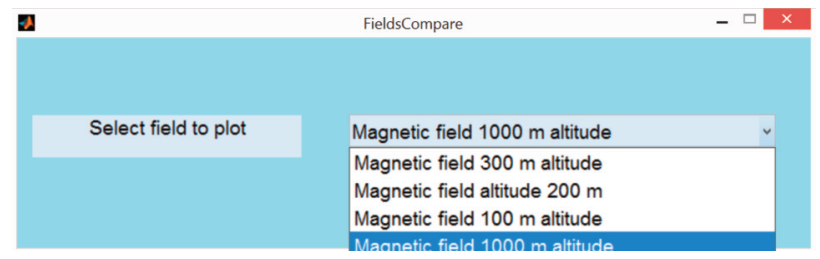

Figure 16. Fields compare module.

pendently and does not perform any check to see if the model is located near the survey area. This can be very annoying because the field can be computed very far from the source area and it can happen especially when a model has been loaded. In the new GaMField version, we have implemented a menu to help the user. Basically, under the menu File -> Survey parameters we have added the menu "Set from Model". The software will automatically modify survey parameters to make the survey area roughly cover the loaded source. The user can further modify the survey parameters if he/she likes.

\subsection{Unify two or more models}

This feature could be very advantageous when the user needs to build two models geographically very far or having very different shapes so that it is inconvenient to use the same ModelBuilder session to realize them. In this case, let's assume we have two or more models for GaMField (produced externally or by using ModelBuilder), we just need to use the menu File -> Model -> Unify two models or the menu File $>$ Model -> Unify more models. The software will automatically produce a single model including both of them.

\subsection{Change whole source magnetic parameters}

It has come out from users that sometimes the source produced needs to be changed in terms of inclination, declination or magnetization. Basically the user wants to change one or more of such parameters without modifying structure geometry. In the new version of GaMField, one can find such feature in ModelBuilder, under the buttons "Change Inclination", "Change Declination" and "Change Magnetization". The software will ask for parameters to be used and a confirmation from the user.

\subsection{Analytical signal}

In the new GaMField version, the Analytical signal computation has been added. The user has not to follow any specific instruction but it will be computed only if the "Compute Gradients" check box is selected. Then the analytical signal could be saved or visualized by means of the two menu View -> Complete Model effects and View -> Complete DEM effects as all other computed components.

\subsection{Fields comparing tool}

A nice tool suggested by many users is the possibility to save different fields computed during the working session and compare them. For example, we would like to compare the field generated by the same model but at different elevations. In this GaMField version we have added the possibility to insert one or more fields into "history" which will be stored during the session. The way to do it is by using menu Special -> Add Field to History, Once this menu has been selected, GaMField asks for a note to label such field. It is very important for the user to choose a note expressing the field details. For example a good note could be "Magnetic Field Altitude $300 \mathrm{~m}$ " as shown in the Figure 15.

If the user wants to compare some stored fields he/she needs to use menu Special -> Compare Fields tool and select the fields he/she wants to compare by means of the combo box shown in Figure 16

\section{Conclusions}

GaMField was freely released for the first time about six years ago and a lot of users have been registered at website http:/ / geosoftware.sci.ingvit to download it. During these years, we have received a lot of 
positive feedback and improvement proposals. This circumstance triggered the effort to release a new version of the software where additional useful tools have been integrated and made available to the scientific community through the same forum.

\section{References}

Al-Rahim, A. M. (2013). Locating of promising stratigraphic traps of pinch-out type in Iraq-new sense. Arabian Journal of Geosciences, 6(5):1381-1393.

Fedi, M. and Rapolla, A. (1999). 3d inversion of gravity and magnetic data with depth resolution. Geophysics, 64:452-460.

Guillen, A. and Menichetti, V. (1984). Gravity and magnetic inversion with minimization of a specific functional. Geophysics, 49:1354-1360.

Guo, Z.-Y., Liu, D.-J., Pan, Q., and Zhang, Y.-Y. (2015). Forward modeling of total magnetic anomaly over a pseudo-2D underground ferromagnetic pipeline. Journal of Applied Geophysics, 113:14-30.

Jeshvaghani, M. S. and Darijani, M (2014). Two-dimensional geomagnetic forward modeling using adaptive finite element method and investigation of the topographic effect. Journal of Applied Geophysics, 105:169-179.

Kim, S.-S. and Wessel, P. (2016). New analytic solutions for modeling vertical gravity gradient anomalies. Geochemistry Geophysics Geosystems, 17(5):19151924.

Last, B. and Kubic, K. (1983). Compact gravity inversion. Geophysics, 48:713- 721.

Li, Y. and Oldenburg, D. (1996). 3-d inversion of magnetic data. Geophysics, 61:394-407.

Liu, S., Hu, X., Xi, Y., and Liu, T. (2015). 2D inverse modeling for potential fields on rugged observation surface using constrained Delaunay triangulation. Computers \& Geosciences, 76:18-30.

Montesinos, F. G., Blanco-Montenegro, I., and Arnoso, J. (2016). Three- dimensional inverse modelling of magnetic anomaly sources based on a genetic algorithm. Physics of The Earth and Planetary Interiors, 253:74-87.

Nicolosi, I., Blanco-Montenegro, I., Pignatelli, A., and Chiappini, M. (2006). Estimating the magnetization direction of crustal structures by means of an equivalent source algorithm. Physics of the Earth and Planetary Interiors, 155:163-169.

Pan, Q., Liu, D.-J., Guo, Z.-Y., Fang, H.-F., and Feng, M.Q. (2016). Magnetic anomaly inversion using magnetic dipole reconstruction based on the pipeline section segmentation method. Journal of Geophysics and Engineering, 13(3):242-258.
Pignatelli, A., Nicolosi, I., Carluccio, R., Chiappini, M., and von Frese, R. (2011). Graphical interactive generation of gravity and magnetic fields. Computers \& Geosciences, 37, issue 4:567-572.

Pignatelli, A., Nicolosi, I., and Chiappini, M. (2007). An alternative 3 -d source inversion of magnetic anomalies method with depth resolution. Annales of Geophysics, 49:1021- 1027.

Speranza, F. and Minelli, L. (2014). Ultra-thick Triassic dolomites control the rupture behavior of the central Apennine seismicity: Evidence from magnetic modeling of the L'Aquila fault zone. Journal Of Geophysical Research-Solid Earth, 119(9):67566770.

Speranza, F., Minelli, L., Pignatelli, A., and Chiappini, M. (2012). The Ionian Sea: The oldest in situ ocean fragment of the world? Journal of Geophysical Research-Solid Earth, 117.

Tontini, F. C. (2012). Rapid interactive modeling of 3D magnetic anomalies. Computers \& Geosciences, 48:308-315.

\footnotetext{
*Corresponding author: Alessandro Pignatelli, Istituto Nazionale di Geofisica e Vulcanologia, Roma, Italy; email: alessandro.pignatelli@ingv.it.
}

C 2018 by the Istituto Nazionale di Geofisica e Vulcanologia. All rights reserved. 